Original paper

\title{
Implementation of a dose calculation algorithm based on Monte Carlo simulations for treatment planning towards MRI guided ion beam therapy
}

\author{
Fatima Padilla-Cabal $^{\mathrm{a}, \mathrm{b}, *}$, Andreas Franz Resch ${ }^{\mathrm{a}, \mathrm{b}}$, Dietmar Georg ${ }^{\mathrm{a}, \mathrm{b}}$, Hermann Fuchs $^{\mathrm{a}, \mathrm{b}}$ \\ ${ }^{\text {a }}$ Department of Radiation Oncology, Medical University of Vienna/AKH, Vienna, Austria \\ ${ }^{\mathrm{b}}$ Christian Doppler Laboratory for Medical Radiation Research for Radiation Oncology, Medical University of Vienna, Vienna, Austria
}

\section{A R T I C L E I N F O}

\section{Keywords:}

Treatment planning

Proton therapy

Magnetic fields

\begin{abstract}
A B S T R A C T
Magnetic resonance guidance in particle therapy has the potential to improve the current performance of clinical workflows. However, the presence of magnetic fields challenges the current algorithms for treatment planning. To ensure proper dose calculations, compensation methods are required to guarantee that the maximum deposited energy of deflected beams lies in the target volume. In addition, proper modifications of the intrinsic dose calculation engines, accounting for magnetic fields, are needed. In this work, an algorithm for proton treatment planning in magnetic fields was implemented in a research treatment planning system (TPS), matRad. Setup-specific look up tables were generated using a validated MC model for a clinical proton beamline (62.4 $215.7 \mathrm{MeV}$ ) interacting with a dipole magnet $(\mathrm{B}=0-1 \mathrm{~T})$. The algorithm was successfully benchmarked against MC simulations in water, showing gamma index $(2 \% / 2 \mathrm{~mm})$ global pass rates higher than $96 \%$ for different plan configurations. Additionally, absorbed depth doses were compared with experimental measurements in water. Differences within $2 \%$ and $3.5 \%$ in the Bragg peak and entrance regions, respectively, were found. Finally, treatment plans were generated and optimized for magnetic field strengths of 0 and $1 \mathrm{~T}$ to assess the performance of the proposed model. Equivalent treatment plans and dose volume histograms were achieved, independently of the magnetic field strength. Differences lower than $1.5 \%$ for plan quality indicators $\left(D_{2} \%, D_{50 \%}\right.$, $\mathrm{D}_{90 \%}, \mathrm{~V}_{95 \%}$ and $\mathrm{V}_{105 \%}$ ) in water, a TG119 phantom and an exemplary prostate patient case were obtained. More complex treatment planning studies are foreseen to establish the limits of applicability of the proposed model.
\end{abstract}

\section{Introduction}

Magnetic Resonance Image (MRI) guidance in external beam therapy potentially allows a precise monitoring of changes in patient anatomy and tumor tracking, while offering an excellent soft-tissue contrast [1]. Integrated MRI radiotherapy units using photons from linear accelerators (MRgXT) are already commercially available [2,3], expecting to improve target dose conformity, better sparing of organs at risk (OAR) and to reduce toxicities [4-8]. In recent years, the use of this technique for precision radiotherapy expanded, due to the demonstrated improvements on treatment accuracy and better tumor delineations [9], combined with the fact that no imaging dose is delivered to the patient. Even more significantly than for photon beam therapy, MRI guidance has the potential to improve the current performance for particle therapy workflows. Better delineation and more precise description of anatomical changes are expected to have a large impact on particle therapy, especially where steep dose gradients are frequently observed. Furthermore, MRgPT online treatment plan adaptation strategies can potentially reduce treatment margins and achieve more conformal dose distributions for high precision treatments of moving targets. Considering that, different studies addressed feasibility aspects for future hybrid MR particle therapy (MRgPT) systems from a technical $[9,10]$ and dosimetric [11-13] point of view. As a result, the first proofof-concept, combining a $0.22 \mathrm{~T}$ open MR with a pencil beam scanning (PBS) delivery system [14] was recently presented.

Besides the technical hurdles of integrating MRI scanners to particle beam lines, current algorithms for treatment planning are severely challenged. The presence of magnetic fields distorts the trajectory of the individual pencil beams, depending on the beam energy, magnetic field strength and the relative orientation between the beam direction and the field lines $[15,16]$. The change on particle trajectories results in different beam spot positions within the patient. Before dose calculations, these changes need to be predicted and corrected in the early stages of treatment planning. Analytical [15-20] and full based Monte

\footnotetext{
*Corresponding author at: Department of Radiation Oncology, Medical University of Vienna/AKH, Vienna, Austria. Christian Doppler Laboratory for Medical Radiation Research for Radiation Oncology, Medical University of Vienna, Vienna, Austria

E-mail address: fatima.padillacabal@meduniwien.ac.at (F. Padilla-Cabal).
} 
Carlo (MC) methods $[11-13,21]$ can be used to estimate the lateral and angular deflection of every single beam path towards and within the patient. However, the respective models describing the interactions between particle beam lines and resulting magnetic field maps for specific MRgPT systems needs to be carefully validated against experimental measurements.

Nowadays, several research groups are exploring the feasibility, robustness and main challenges of intensity modulated MRgPT treatment planning in magnetic fields $[11,12,21,22]$. It was shown that target coverage can be compromised, if compensation methods during beam delivery are not considered $[16,22]$. So far, the common strategy adopted is to split the dose calculations into two main steps: first the adequate selection or adaptation of beam spots to ensure that the maximum deposited energy of deflected beams is located in the target volume, and afterwards the proper modifications of the intrinsic dose calculation algorithm, accounting for magnetic fields [11,12,21,22]. Different methods on beam spot adaptation for laterally deflected proton beams in transverse magnetic fields were suggested [12,15-18], considering angular, energy or lateral shifting corrections. Furthermore [22], machine and patient-specific corrections were recently proposed for protons in inline MRI fringe fields by means of single angle rotations and radial scaling factors, calculated for different beam energies and magnetic field strengths. The dosimetric feasibility of MRgPT using patient case data was also demonstrated [11,12,21,22]. Dose calculations encompassing clinical or generic beam lines and either homogeneous or "realistic" magnetic field maps were performed using full MC-based algorithms.

In particle therapy, most widely used commercial TPS such as Raysearch (RaySearch Medical Laboratories AB, Stockholm, Sweden), Eclipse (Varian Medical Systems, Palo Alto, CA, USA), XiO (Elekta AB, Stockholm, Sweden) or Syngo RT (Siemens, Erlangen, Germany) employ semi-analytical pencil beams algorithms for dose calculation. In addition, new MC based engines are becoming available in some TPS and being commissioned for clinical practice $[23,24]$. To the best of our knowledge, neither of the commercially available TPS incorporate dose calculation methods for particles in magnetic fields.

In this work, we propose a procedure for treatment planning of protons in transverse magnetic fields, based on a custom pencil beam algorithm (PBA) and a compensation method for beam delivery. The algorithm was implemented and tested in the open-source research TPS, matRad. MC simulations, using the GATE/Geant4 package, were employed to support several aspects of treatment planning, and to generate calibration and validation data for the model.

\section{Materials and methods}

\subsection{Treatment planning and dose calculation}

Treatment planning studies for protons in magnetic fields were done using the open-source research TPS, matRad [25]. The implemented general model for treatment planning, starting from the patient anatomical model to final plan optimization, was followed. However, dose calculations were performed using a custom algorithm to properly describe the effect of magnetic fields on each individual pencil beam. A new compensation method for the treatment plan delivery when magnetic fields are applied was also incorporated, as depicted in Fig. 1.

For charged particles, dose depositions are calculated in matRad using a conventional dose-to-water-pencil beam algorithm [26] based on look-up tables (LUT). Water equivalent lengths $z_{e q}$ are determined from the material stopping power ratios to water [25]. For each pencil beam, the dose is determined as the product of the longitudinal depthdependent deposition function $E_{\text {dep }}\left(z_{\text {eq }}\right)$ and a lateral component $f(x, y)$ as described in Eq. (1).

$D_{k}=E_{\text {dep }}\left(z_{\text {eq }}\right) \cdot f(x, y)$

where the two-dimensional lateral component $f(x, y)$ is calculated

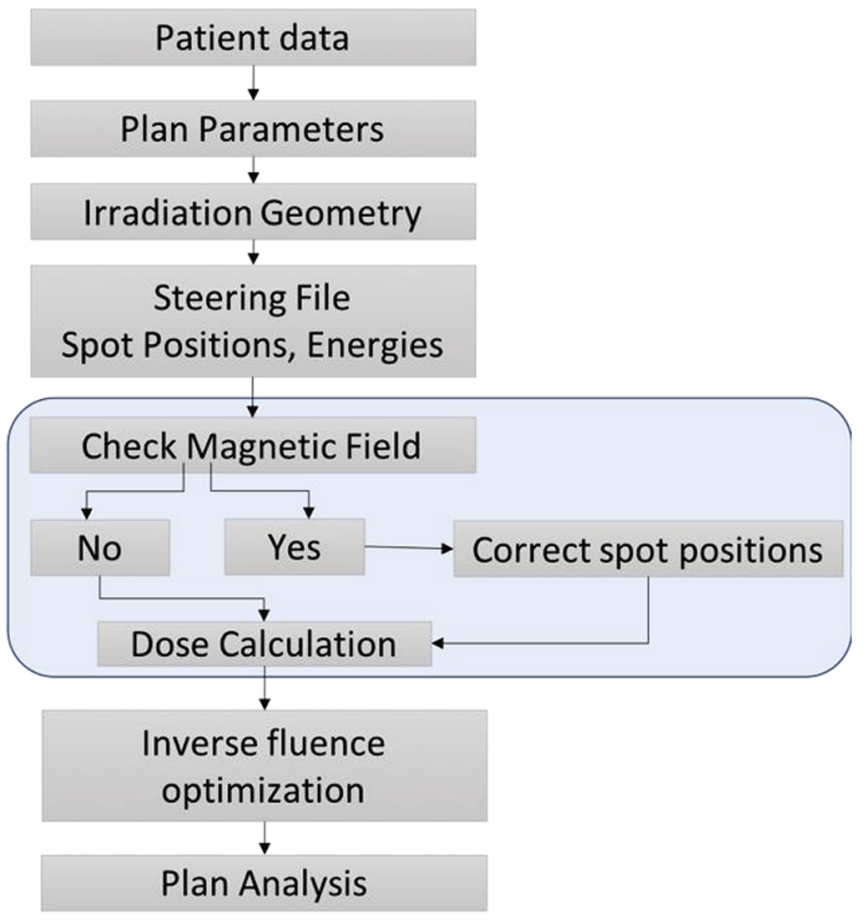

Fig. 1. Flowchart of the matRad treatment planning workflow used through this work. The modifications to include the magnetic field effects on dose calculations and beam delivery are highlighted in light blue. (For interpretation of the references to colour in this figure legend, the reader is referred to the web version of this article.)

using a symmetric rotation of a beam profile, described either by a single or by a superposition of two Gaussian functions[25].

In our calculations, a similar approach was followed, but generic LUT were replaced with custom base data files. A validated Monte Carlo (MC) beam model [27], encompassing a complete in-house established proton beam line model $[28,29]$ and a three-dimensional magnetic field map, was employed to generate the input LUT for the algorithm, see Fig. 2. The MC model was used to predict three-dimensional dose distributions in water, from where both $E_{d e p}^{B}\left(z_{e q}\right)$ and $f^{B}(x, y)$ functions were deducted. The two-dimensional lateral component $f^{B}(x, y)$ was described by a combination of individual probability density functions for both individual coordinates $f_{x}^{B}(x), f_{y}^{B}(y)$ scored at each penetration depths.

\subsection{Generation of input calibration data}

A clinical proton pencil beam scanning (PBS) system from the MedAustron ion beam therapy center was simulated interacting with magnetic fields up to $1 \mathrm{~T}$ using an extended version of the GATE8.0/

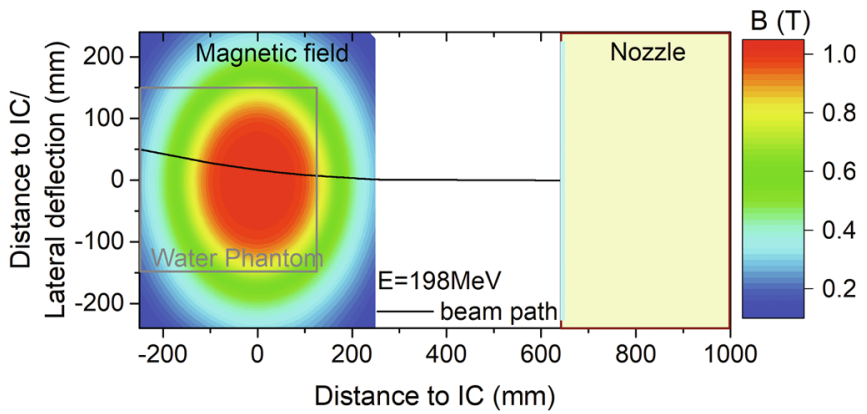

Fig. 2. Schematic overview of the MC beam model used to generate the input calibration data of the dose calculation algorithm. 
Geant4.10.03.p03 toolkit. Details on the magnetic field and physics models, as well as simulation parameters were published earlier $[27,28,30-33]$. Electromagnetic and hadronic processes were described using the QBBC_EMZ physics list, while material compositions and ionization potential were obtained from the NIST-PSTAR database [34]. A water phantom of $400 \times 400 \times 400 \mathrm{~mm}^{3}$ was placed at a source surface distance (SDD) of $522 \mathrm{~mm}$ from the Nozzle exit, assuring that the phantom was located in the highest homogeneous region of the magnetic field, see Fig. 2. Proton beams with 14 different energies ranging from 62.4 to $215.7 \mathrm{MeV}$, corresponding to beam ranges in water from 30 to $290 \mathrm{~mm}$, were employed. Dose distributions were scored in cubic voxels of $2 \times 2 \times 2 \mathrm{~mm}^{3}$ covering the full phantom volume for magnetic field intensities of 0 and $1 \mathrm{~T}$. Additionally, laterally integrated dose distributions as a function of depth (IDD) were scored using voxels of $400 \times 400 \times 0.1 \mathrm{~mm}^{3}$ for the above-mentioned configurations. For the sake of sufficient counting statistics, a total number of $10^{7}$ primary particles were simulated for each of the considered 14 initial beam energies and the two magnetic field intensities.

At MedAustron, a library of 255 energies is clinically used for proton beams, fulfilling the treatment planning requirements of placing Bragg peaks between 30 and $380 \mathrm{~mm}$ water-equivalent (WE) depths with a spacing between 1 and $2 \mathrm{~mm}$. The same compromise was adopted in the generation of our calibration data, although the maximal energy was limited to $215.7 \mathrm{MeV}$, corresponding to a range in water of $290 \mathrm{~mm}$. Due to constraints in the magnetic field homogeneity region, restricted to a diameter of $150 \mathrm{~mm}$, only energies corresponding to ranges in water lower than $290 \mathrm{~mm}$ were considered to ensure dose calculations in equivalent magnetic fields. As result, a calibration library of 210 energies (62.4-215.7 MeV) was built and used as input data for treatment planning.

For the pencil beam algorithm, $E_{d e p}^{B}\left(z_{e q}\right)$ functions were obtained directly from the IDD distributions simulated with GATE with and without magnetic fields. New IDD functions were linearly interpolated from the simulated data, including all the 210 calibration energies required for treatment planning. The ranges R80 were determined for all the energies at a dose level of $80 \%$ in the fall-off region.

\subsubsection{Lateral beam parametrization model}

According to Eq. (1), the precision on dose calculations relies also on the accuracy of the models used for the lateral characterization $f^{B}(x, y)$ of proton pencil-like beams. To calculate this function, simulated beam spots were analyzed at each depth and lateral profiles for both $x$ and $y$ coordinates were extracted. A parametrization method, based on the direct fitting of both MC simulated profiles using a double Gaussian function [35] was chosen for each individual coordinate, as shown in Eq. (2).

$$
\begin{aligned}
& f_{x}^{B}(x)=\left(1-w_{x}\left(E, z_{e q}\right)\right) \cdot G_{1 x}(x)+w_{x}\left(E, z_{e q}\right) \cdot G_{2 x}(x) \\
& f_{y}^{B}(y)=\left(1-w_{y}\left(E, z_{e q}\right)\right) \cdot G_{1 y}(y)+w_{y}\left(E, z_{e q}\right) \cdot G_{2 y}(y) \\
& G_{1,2 x}(x)=\frac{1}{\sqrt{\pi} \sigma_{1,2 x}\left(E, z_{e q}\right)} \exp \left[-\frac{x^{2}}{\sigma_{1,2 x}^{2}\left(E, z_{e q}\right)}\right] \\
& G_{1,2 y}(x)=\frac{1}{\sqrt{\pi} \sigma_{1,2 y}\left(E, z_{e q}\right)} \exp \left[-\frac{\left(y-\mu\left(E, z_{e q}\right)\right)^{2}}{\sigma_{1,2 y}{ }^{2}\left(E, z_{e q}\right)}\right]
\end{aligned}
$$

A fitting procedure was implemented in MATLAB_R2017b (The MathWorks Inc., USA) and the central coordinate of the deflected beam $\mu\left(E, z_{\text {eq }}\right)$, the corresponding $\sigma_{1,2}\left(E, z_{e q}\right)$ for the narrow and broader Gaussians, as well as the ratio $w\left(E, z_{e q}\right)$ of the corresponding areas between both Gaussians were tabulated for the 14 simulated energies at each depth. Due to the influence of the transverse magnetic fields, different lateral dose profiles were obtained for both coordinates. Therefore, the parameters were stored independently for the deflected coordinate $(y)$ and the non-deflected coordinate $(x)$. The two-dimensional probability density function was hence calculated as:

$f^{B}(x, y)=f_{x}^{B}(x) \cdot f_{y}^{B}(y)$

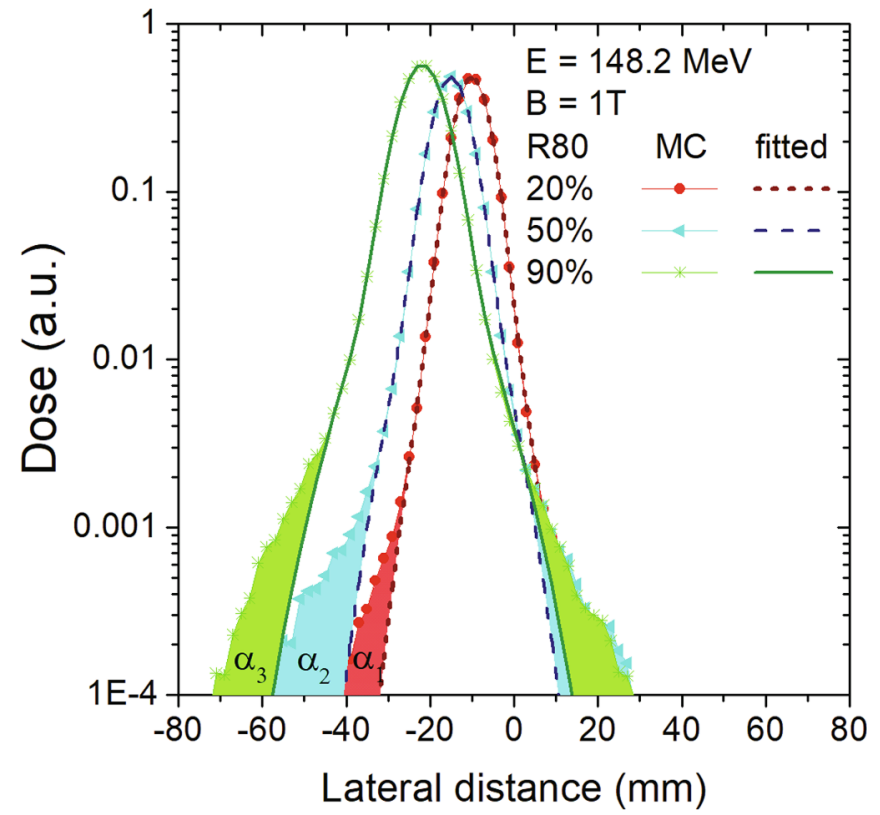

Fig. 3. Fitted and simulated lateral dose profiles for $148.2 \mathrm{MeV}$ protons in a magnetic field of $B=1 \mathrm{~T}$ scored at penetration depths of 20,50 and $90 \%$ of the $\mathrm{R} 80$. The shaded regions represent the underestimated doses using the parametrization method.

assuming independence between the variables $x, y$.

Dose contributions at lateral distances of up to $200 \mathrm{~mm}$ were accounted to estimate the probability density functions $f_{x}^{B}(x), f_{y}^{B}(y)$, including both the core and halo dose regions [36]. For proton beams, the double Gaussian fitting model agreed well with the central beam component but underestimated lateral doses at farther lateral distances from the center, as shown in Fig. 3. Those small dose differences $\left(<0.1 \%\right.$ of $\left.\mathrm{D}_{\max }\right)$ integrated over a large lateral area sum up to a nonnegligible $15 \%$ contribution to the total energy deposited, as observed from Fig. 3. Therefore, IDD were rescaled by a correction factor $\alpha\left(E, z_{\text {eq }}\right)$. Finally, the dose contribution of each single pencil beam was obtained as:

$D_{k}=\alpha\left(E, z_{e q}\right) \cdot E_{d e p}^{B}\left(z_{e q}\right) \cdot f_{x}^{B}(x) \cdot f_{y}^{B}(y)$

The accuracy of the parametrization method was tested for single beams $(62.4,148.2$ and $215.7 \mathrm{MeV})$. Predicted doses from spots located at penetration depths of 20,50 and $90 \%$ of the R80 range were compared with MC simulations. Finally, all 7 parameters $\mu\left(E, z_{\text {eq }}\right)$, $\sigma_{1,2, x, y}\left(E, z_{e q}\right), w_{x, y}\left(E, z_{e q}\right)$ and the $\alpha\left(E, z_{e q}\right)$ were linearly interpolated from the simulated data to the 210 calibration energies look-up tables.

\subsection{Treatment plan adaptation in magnetic fields}

In addition to the new dose calculation engine, a compensation method for beam delivery in magnetic fields was implemented. In matRad, the geometry for irradiation for every beam orientation (spot positions and energy layers) is selected using a ray tracing algorithm accounting for the patient geometry and the projection of the target volume onto the beam's eye view [25]. Individual pristine pencil beams are placed equidistantly in coordinates $\left[X_{i}, Y_{i}\right]$ with energies $E_{i}$ to cover the entire target volume [25].

The change on the pristine pencil beams paths in our setup-specific configuration due to the impact of magnetic fields, was described using the same validated MC model. For deflected beams, lateral beam coordinates were extracted from the parametrized datasets at each depth for each individual calibration energy. For each energy layer, the expected bending at the Bragg peak position, $\mu_{R 80}\left(E_{i}\right)$ was then used to predict the projected spot positions (in beams eye view) of deflected 
beamlets in or around the target volume.

To ensure that beamlets were correctly located in the target volume when magnetic fields are applied, the initial spot positions calculated after ray tracing were shifted for each energy layer in the same amount and opposite direction of the expected $\mu_{R 80}\left(E_{i}\right)$. New "adapted" spot positions from the TP were then relocated at $\left[X_{i}, Y_{i}-\mu_{R 80}\left(E_{\max }\right)\right]$, being $\mu_{R 80}\left(E_{\max }\right)$ the lateral deflection expected for the maximum energy of the beamlets. Additionally, a "virtual" planning target volume (PTV) was defined, adding a lateral safety margin $\delta_{y}=\mu_{R 80}\left(E_{\max }\right)-\mu_{R 80}\left(E_{\min }\right)$ to the PTV. Those safety margins comprised the differences between the projected spot positions expected for the minimum and maximum energy layers used for full target coverage. The effectiveness of the method was assessed, comparing dose distributions for cubic targets $\left(40 \times 40 \times 40 \mathrm{~mm}^{3}\right)$ centered at $60,100,140$ and $180 \mathrm{~mm}$ calculated without and with magnetic fields by means of their resulting dose volume histograms (DVH).

\subsection{Evaluation of the implemented algorithm}

In a first stage the new method for proton treatment planning in magnetic fields was validated against MC simulations in water. Single field treatment plans (TP) yielding a box shaped target SOBP $\left(40 \times 40 \times 40 \mathrm{~mm}^{3}\right)$ centered at depths of $60,100,140$ and $180 \mathrm{~mm}$ and magnetic field strengths of 0 and $1 \mathrm{~T}$ were generated in matRad. The plans were recalculated in GATE, using the same configuration of spot positions, energies and weights, as existing in the TPS. Tridimensional dose distributions were scored in a voxel grid of $2 \times 2 \times 2 \mathrm{~mm}^{3}$ overall a $500 \times 500 \times 400 \mathrm{~mm}^{3}$ homogeneous water phantom. The same SDD $=522 \mathrm{~mm}$ was employed in the validation simulations, to ensure same beam modelling conditions, as used for the generation of the input calibration data for the TPS. For each treatment plan, $2 \mathrm{e} 7$ primary events were simulated to achieve resulting statistical uncertainties in the order of $1 \%$. A $\gamma$-index analysis using a $2 \% / 2 \mathrm{~mm}$ criteria was proposed to compare dose maps computed with matRad and recalculated with GATE, applying a global dose threshold of $1 \%$ of the prescribed dose.

\subsubsection{Experimental measurements}

In addition to the MC re-calculation, absorbed dose measurements were conducted to test the accuracy of the calculation model. Proton beams with energies ranging from 62.4 to $215.6 \mathrm{MeV}$ were deflected by the resulting magnetic field from a dipole research magnet (Danfysik A/ $\mathrm{S}$, Taastrup, Denmark) placed in the room isocenter. Experiments were conducted using magnetic field strengths of $\mathrm{B}=0 \mathrm{~T}$ and $\mathrm{B}=1 \mathrm{~T}$, perpendicular to the incident beam direction. The use of conventional dosimetric equipment was limited due to the spatial limitations between the magnet poles $(135 \mathrm{~mm})$ and the magnetic field homogeneity region, constrained to a sphere-shaped volume of $75 \mathrm{~mm}$ radius around the center of the magnet. An in-house designed water phantom of $814 \times 402 \times 110 \mathrm{~mm}^{3}$ was utilized to perform depth dose measurements within the magnet. The detector was mounted on a remotecontrolled moving mechanism, with a depth step precision of $1 \mathrm{~mm}$. Absorbed dose was measured at different penetration depths using a Roos chamber (PTW type 34001) connected to a UNIDOS electrometer (PTW, Freiburg, Germany), cross-calibrated against a Farmer chamber at $\mathrm{B}=0$. A closer view to the experimental setup used through our measurements is shown in Fig. 4.

Treatment plans were calculated and optimized in matRad for a $40 \times 40 \times 40 \mathrm{~mm}^{3}$ target centered at $140 \mathrm{~mm}$ from the phantom surface, for magnetic field strengths of 0 and $1 \mathrm{~T}$. For plan irradiation, a custom interface was developed to convert the exported matRad treatment plans to a readable format, as accepted for the accelerator. Absorbed doses were measured with the Roos chamber of $16 \mathrm{~mm}$ diameter active volume centered in the homogeneous dose distribution region, more than $10 \mathrm{~mm}$ away from the beginning of the lateral dose fall-off region. To warrant a fair comparison with measured dose values, TPS and GATE calculated doses were volume averaged in the voxels corresponding to the effective volume of the Roos chamber.

\subsection{Pilot treatment planning studies in magnetic fields}

The proposed model for dose calculation was employed to assess the impact of transverse magnetic fields on treatment planning. Two anonymized open-source patient/phantom datasets, included into the public matRad's code base [25] were chosen to compare the plan quality parameters for magnetic field strengths of 0 and $1 \mathrm{~T}$. Treatment plans were calculated for a TG119 phantom and a prostate patient case, using patient data (CT images and segmentation structures) from the CORT dataset [37]. Details about the plans specifications, as well as the dose/volume constraints for the targets and organs at risk (OAR) are summarized in Table 1 . Plans were calculated using beam spot distances of 3-5 mm, and fluence-optimized using a dose influence matrix concept and a constant relative biological effectiveness (RBE) for protons of 1.1. As implemented in matRad, optimization parameters (penalties, objectives, constraints) were selected to fulfill dose distributions requirements, as recommended in the clinics. To evaluate only the performance of the algorithm for magnetic fields, the same plan parameters and optimization conditions were employed for magnetic field strengths of 0 and $1 \mathrm{~T}$. Dose distributions were compared for both generated plans from the observed differences between them, as well as the DVH parameters.

\section{Results}

\subsection{MC generated input data for the TPS}

A representative subset of the 210 IDD distributions required for treatment planning are displayed in Fig. 5a. The overall depths covered by the energy library as well as the retraction on the Bragg peak (BP) positions due to the influence of the magnetic field are also shown in Fig. 5a. The average separation of consecutive BP positions were $1.00 \pm 0.07 \mathrm{~mm}$ and $2.00 \pm 0.10 \mathrm{~mm}$ for $\mathrm{B}=0 \mathrm{~T}$ and $1.00 \pm 0.07 \mathrm{~mm}$ and $1.98 \pm 0.09 \mathrm{~mm}$ for $\mathrm{B}=1 \mathrm{~T}$, showing a good agreement with the requirements for treatment planning. Moreover, the lateral displacement parameters $\mu\left(E, z_{\text {eq }}\right)$ are displayed in Fig. $5 \mathrm{~b}$. Lateral deflections ranged from 7.2 to $13.7 \mathrm{~mm}$ at the entrance of the phantom, and from 17.0 to $37.1 \mathrm{~mm}$ at the Bragg peak of the highest calibration energy, 215.7 MeV (Table 2).

\subsection{Evaluation of the implemented algorithm}

TPs generated in matRad for a $40 \times 40 \times 40 \mathrm{~mm}^{3}$ cubic target centered at distances $60-180 \mathrm{~mm}$ using magnetic field strengths of 0 and $1 \mathrm{~T}$ showed equivalent dose distributions in the target volume. Comparable DVH were achieved and non-significant deviations using a student $t$-test (p-value 0.05 ) for all plan quality parameters were observed at the target volume.

Good agreement was obtained between dose distributions from the TPS and MC simulations. An example of the dose map scored at the isocenter plane for a $40 \times 40 \times 40 \mathrm{~mm}^{3}$ SOBP centered at $100 \mathrm{~mm}$ is shown in Fig. 6, displaying the central bidimensional dose distributions calculated with the proposed algorithm and recalculated with GATE in water, as well as the gamma-index map using a $(2 \% / 2 \mathrm{~mm})$ criteria. Comparable level of agreement was achieved for all the analyzed configurations using either single beams, or cubic targets. A summary of the results is presented in Table 3, demonstrating good performance of the algorithm for a wide range of energies $(72.4-188.7 \mathrm{MeV})$ with and without magnetic field.

Results of the experimental benchmarking of the proposed dose calculation algorithm are shown in Fig. 7. Absorbed depth dose measurements for a $40 \times 40 \times 40 \mathrm{~mm}^{3}$ SOBP centered at $140 \mathrm{~mm}$ are compared with TPS and MC calculations for magnetic fields strengths of 


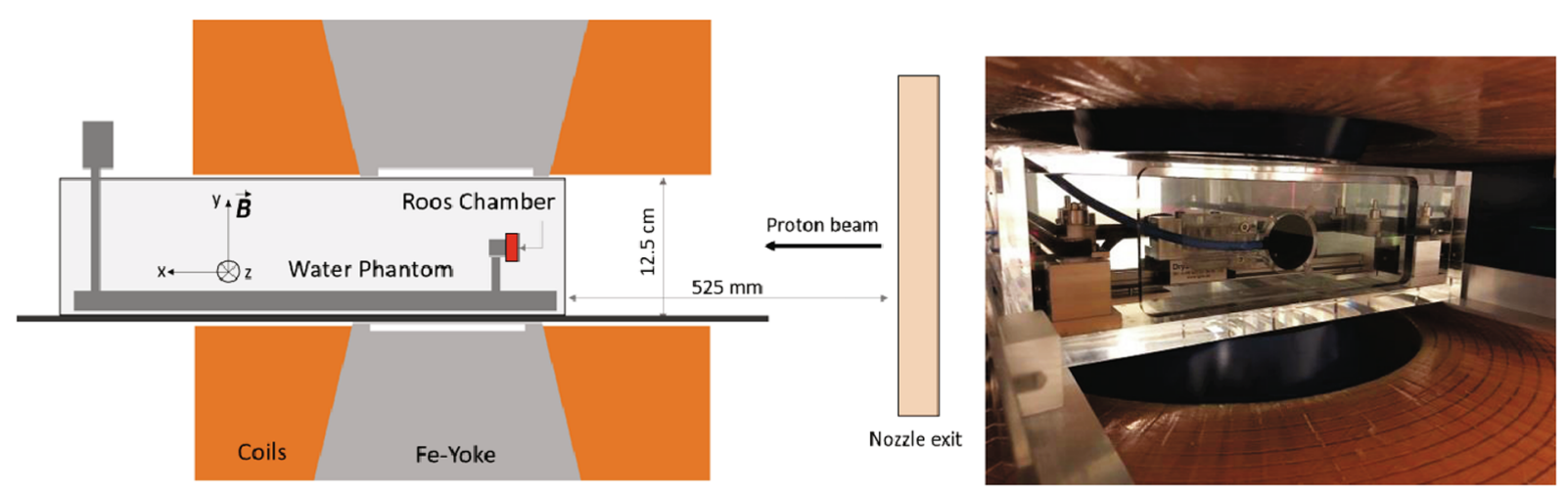

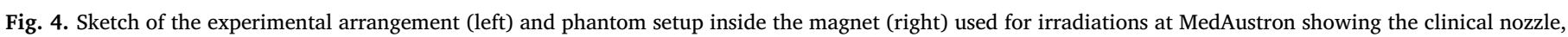
the dipole research magnet and the water phantom used for dosimetric measurements.

Table 1

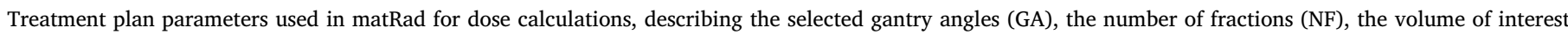
(VOI) and the prescribed doses and objectives for optimization.

\begin{tabular}{|c|c|c|c|c|c|c|}
\hline Patient Case & GA (deg) & $\mathrm{NF}$ & VOI & VOI Type & Dose objective & Dose (Gy) \\
\hline \multirow[t]{3}{*}{ TG119 Phantom } & 90,270 & 30 & Outer Target & Target & Sq. deviation & 50 \\
\hline & & & Core & OAR & Sq. overdosing & 25 \\
\hline & & & Body & OAR & Sq. overdosing & 30 \\
\hline \multirow[t]{4}{*}{ Prostate } & 90,270 & 20 & PTV & Target & Sq. deviation & 68 \\
\hline & & & Bladder & OAR & Sq. overdosing & 50 \\
\hline & & & Rectum & OAR & Sq. overdosing & 50 \\
\hline & & & Body & OAR & Sq. overdosing & 30 \\
\hline
\end{tabular}

0 and $1 \mathrm{~T}$. Relative deviations are indicated in the right axes of the plot, demonstrating local deviations in the Bragg peak region in the order of $\pm 2 \%$ from GATE and TPS predictions. Higher discrepancies in the entrance region (up to 4\%) were spotted for the TPS calculations using the LUT for the non-field case.

\subsection{Pilot treatment planning studies in magnetic fields}

The effect of magnetic fields on the treatment plan of the prostate patient case is shown in Fig. 8. A detailed overview of the dose distributions on the axial, coronal and sagittal planes is presented for both magnetic field configurations, as well as their resulting differences. An equivalent target dose conformation was achieved for both cases, using the presented TP method. However, higher doses are delivered to some volumes of the surrounding tissue.

The quality of the treatment plans "adapted" for delivery in presence of a transverse magnetic field of $B=1$ T is presented in Fig. 9 and Table 4. The plan quality indicators $\left(D_{2 \%}, D_{50 \%}, D_{90 \%}, V_{95 \%}\right.$ and $\left.V_{105 \%}\right)$ differed in less than $1.5 \%$ for the prostate patient case and $0.6 \%$ for the phantom case at the target volume. The target dose coverage $V_{95 \%}$ was found to be equivalent for the prostate patient and phantom cases.

\section{Discussion}

A new algorithm for treatment planning in transverse magnetic fields was proposed, based on pencil beam kernels and a compensation method for beam delivery. Dose calculations and plan optimization were performed using a similar approach, as currently used in commercial TPS for particle therapy.

Despite the fact that pencil beam algorithms are not optimal for describing accurately the dose deposition in interfaces of heterogeneity materials [18,38-40], their use for dose calculation and inverse plan optimization is still widespread in clinical practice, due to their faster calculation times and efficient clinical workflows [41]. As previously studied $[13,15]$, transverse magnetic fields modify in-depth and lateral dose contributions of individual pencil beams. Therefore, proper modifications for both functions were required to ensure an accurate dose estimation method. Particularly, a new parametrization model was needed, to describe the characteristic deformations of the lateral profiles encountered in the presence of magnetic fields. Different parametrization models by means of direct fitting of MC lateral profiles are discussed for particle therapy [42-45], being the combination of multiple Gaussians the most frequently used in commercial TPS [40,46-48]. Through this work, a double Gaussian model [35] was preferred, providing acceptable level of accuracy and calculation times. Multiple Coulomb scattering processes of the protons were described in the central Gaussian dose core, while $\alpha\left(E, z_{\text {eq }}\right)$ coefficients were determined to correct the laterally integrated in-depth dose distributions for nuclear and single large-angle Coulomb scattering. A limitation for our model is that the lateral distances considered during the parametrization are higher than the typical radial distances used during treatment planning. As consequence, the amplitude/area ratio for the fitted functions was affected by the farther dose contributions, leading to errors on dose calculations up to $6 \%$ in the entrance and $15 \%$ on the Bragg peak regions for the highest energy of $215.7 \mathrm{MeV}$, if not corrected. A simple method to overcome this effect, was the introduction of the $\alpha\left(E, z_{e q}\right)$ coefficients. Another approach is to introduce a third Gaussian during the parametrization to represent the out-field doses, requiring a higher number of free parameters and increasing calculation times. An alternative parametrization model using a exponentially tailed Gaussian model [18] was also evaluated in the initial phase of this work. A better level of accuracy for fitting of lateral profiles was achieved at a cost of increasing the total number of free parameters from 5 to 7 and consequently, calculation times. Another encountered challenge for dose calculations in transverse magnetic fields, was the non-symmetry observed between the lateral profiles for the deflected and the non-deflected coordinates. Therefore, a radial dose scoring approach, as implemented in most of research TPS was not possible. Instead, a model describing independently and simultaneously both profiles was suggested. Most of the pre-implemented functions in matRad for the determination of lateral dose cutoff, pixel doses and ray tracing were modified accordingly to account for the deflected 

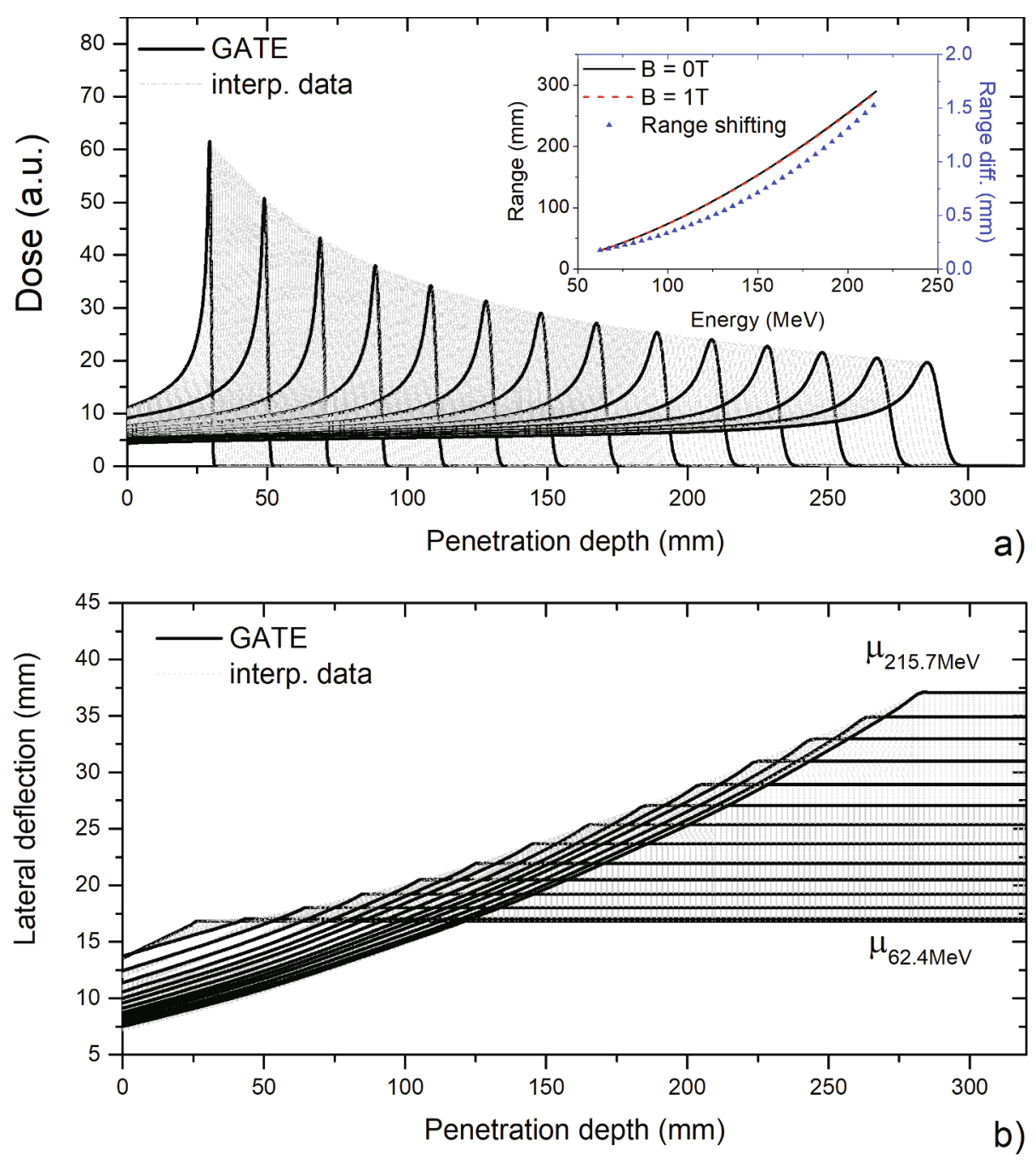

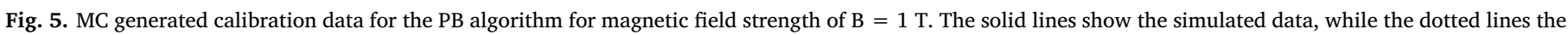

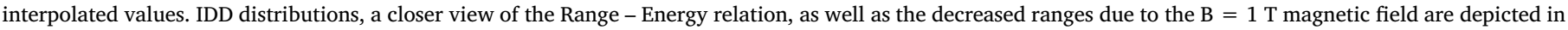
(a). The predicted lateral bending of each pencil beam in a magnetic field of $\mathrm{B}=1 \mathrm{~T}$ is shown in (b).

Table 2

Evaluation of the accuracy of the parametrization model used to estimate the lateral contribution of proton pencil beam doses. Results are shown for both not-rescaled $(\alpha=1)$ and rescaled IDDs.

\begin{tabular}{|c|c|c|c|c|c|c|c|}
\hline \multirow{3}{*}{$\begin{array}{l}\text { Energy } \\
(\mathrm{MeV})\end{array}$} & \multirow[t]{3}{*}{$\mathrm{B}(\mathrm{T})$} & \multicolumn{6}{|c|}{ Gamma Passing Rate $(2 \%, 2 \mathrm{~mm})$} \\
\hline & & \multicolumn{2}{|c|}{ Spot @ 20\% R80 } & \multicolumn{2}{|c|}{ Spot @ 50\% R80 } & \multicolumn{2}{|c|}{ Spot @ 90\% R80 } \\
\hline & & $\alpha=1$ & $\alpha\left(E, z_{\text {eq }}\right)$ & $\alpha=1$ & $\alpha\left(E, z_{e q}\right)$ & $\alpha=1$ & $\alpha\left(E, z_{e q}\right)$ \\
\hline \multirow[t]{2}{*}{62.4} & 0 & 85 & 100 & 84 & 100 & 86 & 100 \\
\hline & 1 & 84 & 100 & 84 & 100 & 84 & 100 \\
\hline \multirow[t]{2}{*}{148.2} & 0 & 79 & 100 & 74 & 100 & 74 & 100 \\
\hline & 1 & 79 & 100 & 72 & 100 & 72 & 100 \\
\hline \multirow[t]{2}{*}{215.7} & 0 & 77 & 100 & 59 & 100 & 67 & 100 \\
\hline & 1 & 78 & 100 & 57 & 100 & 69 & 100 \\
\hline
\end{tabular}

trajectory of the beams.

The accuracy of the dose calculation method presented through this work, is limited by the intrinsic performance of PBA-based dose calculation models. For example, it is well-known that PBA fail to handle incident beams at oblique angles as well as heterogeneities [24,41], as the transport of each individual pencil beam is done considering consecutive slabs of homogeneous materials. To increase the accuracy of PBA-based dose estimations, lateral splitting techniques $[18,49]$ are commonly implemented in commercial TPS, achieving a desired dosimetric precision within reasonable calculation times. However, dose calculations in regions with large tissue heterogeneities [24,41] demonstrated higher discrepancies between PBA and MC-based recalculations. For such clinical scenarios improved dose calculation accuracy can be obtained using MC-based dose engines. Hitherto, most of the commercially available TPS use semi-analytical algorithms based on proton pencil beams for dose calculation. However, dedicated calculation engines based on MC methods are starting to become more available in the clinical practice $[23,24,50]$. Likewise, independent MC dose calculation algorithms $[29,51,52]$ based on general particle transport codes like Geant4 [53] or FLUKA [54] represent attractive solutions to support treatment planning and QA in ion beam therapy. For future MRPT systems, we believe that the combination of fast PBA with complementary MC dose calculation engines will provide a suitable environment mixing accurate physics for modelling the transport of particles in magnetic fields, while keeping the calculation times suitable for typical clinical workflows.

For future MRgPT systems, compensation methods during beam delivery are needed to ensure that the beamlets, after being deflected by magnetic fields, reach the target volume [10,22]. In this work, the impact of the magnetic fields on the projected spot positions at the target volume was predicted using MC simulations in water for a setupspecific configuration (clinical beam + magnetic field map + SSD). 

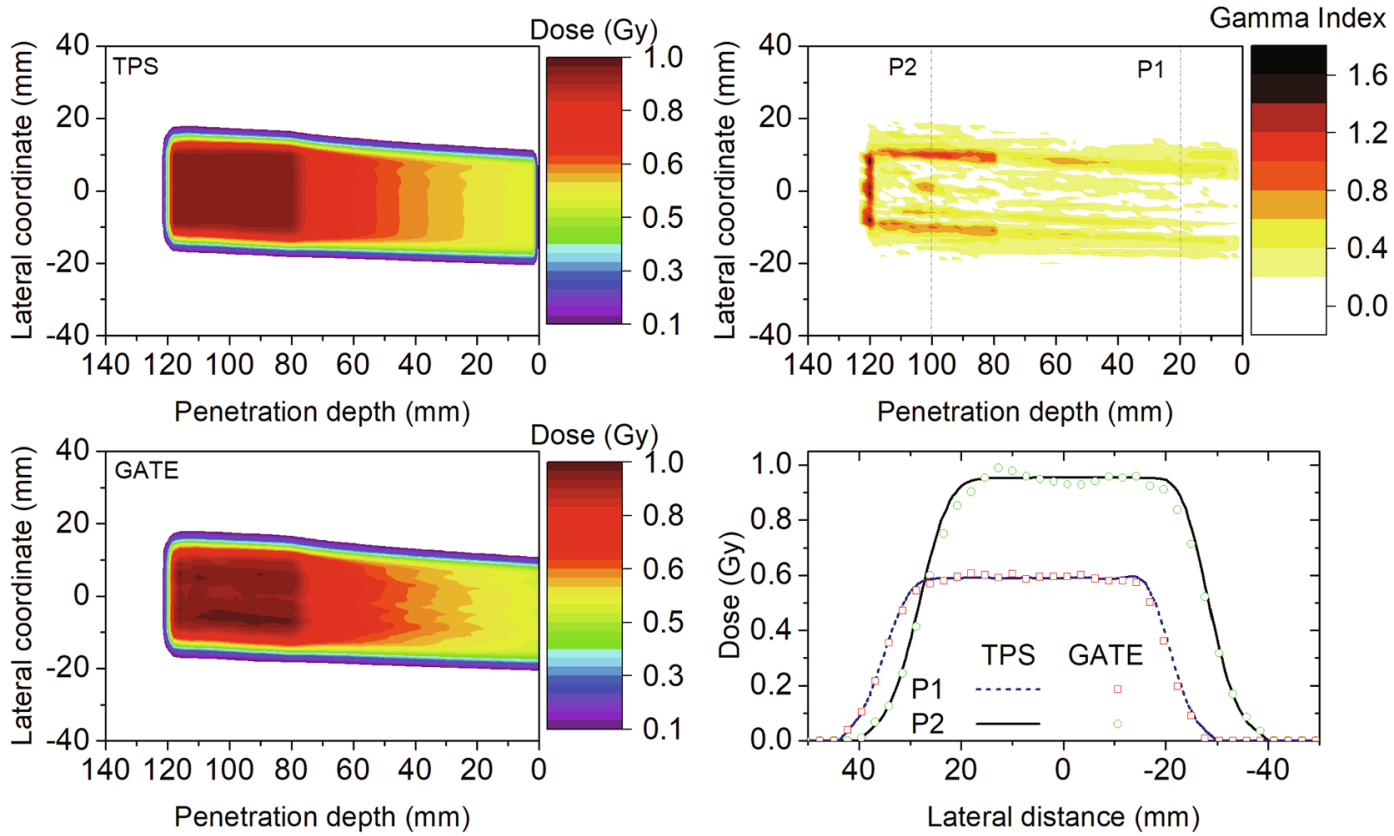

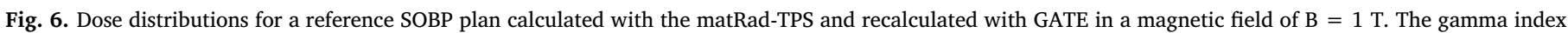

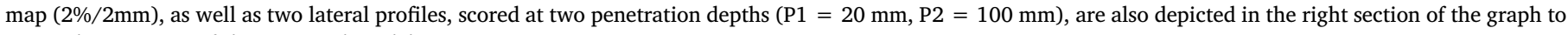
assess the accuracy of the proposed model.

The proposed compensation method using a lateral shifting of the initial spots positions was found to be sufficient to restore the target volume coverage for simple examples in a water and TG119 calibration phantom, covering water equivalent depths in the range of $40-200 \mathrm{~mm}$. Furthermore, for the prostate patient case comparable TPs were also accomplished. However, for more complex treatment plans, like present for intensity modulated MRPT, a simple shifting of the spot positions is foreseen to be insufficient, especially when obliques fields are considered. Instead, either an adjustment of the beam ray tracing algorithm is necessary, or new setup-specific LUT are required to select beamlets whose deflected Bragg peaks are located inside the target. Different semi-analytical $[17,18]$ and full MC models $[11,16,22]$ are currently investigated to be incorporated to the matRad TPS in order to decrease current calculation times required for inverse treatment optimization.

The implementation of the dose calculation algorithm was benchmarked against MC simulations. The observed deviations between measured and calculated plans $(\sim 2 \%)$, as well as the range deviations $(<0.3 \mathrm{~mm})$ and gamma index $(2 \% / 2 \mathrm{~mm})$ mean values $(<0.45)$ and global pass rates (greater than $96 \%$ ), demonstrate an overall good performance of the method. The MC model used through this work was previously validated against experimental measurements [27], representing a reliable independent dose calculation method. In addition, good agreement of TPS and MC beam models was also obtained with experimental data for absorbed doses using a cross-calibrated Roos

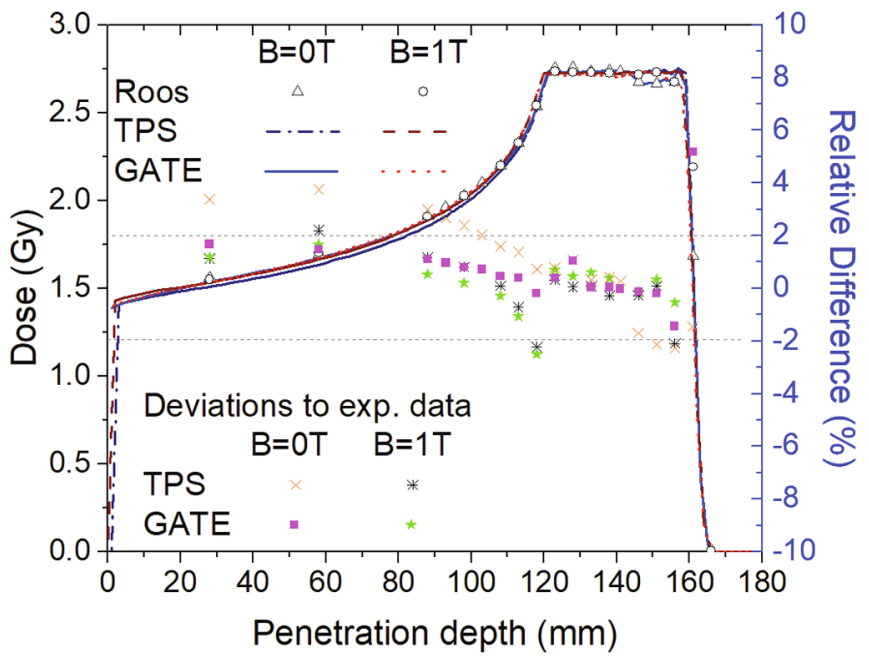

Fig. 7. Comparison between absorbed depth dose distributions for a $40 \times 40 \times 40 \mathrm{~mm}^{3}$ SOBP centered at $140 \mathrm{~mm}$, calculated with the TPS and GATE and measured with a Roos chamber for magnetic fields strengths of $\mathrm{B}=0 \mathrm{~T}$ and $\mathrm{B}=1 \mathrm{~T}$. The relative deviations between the measured and calculated values are also given in the right axes. The limits for $2 \%$ are indicated with grey dotted lines, for a better visualization of the data.

Table 3

Comparison between tridimensional dose distributions from the TPS and recalculated plans with GATE. Difference of the ranges R80 are given, as well as the gamma index $(2 \% / 2 \mathrm{~mm})$ mean values and global pass rates.

\begin{tabular}{|c|c|c|c|c|c|c|c|}
\hline \multirow[t]{2}{*}{ Field } & \multirow[t]{2}{*}{ Energy (MeV) } & \multicolumn{2}{|c|}{ Range diff } & \multicolumn{2}{|c|}{$\gamma$-pass rate $(2 \%, 2 \mathrm{~mm})$} & \multicolumn{2}{|l|}{$\gamma$-mean } \\
\hline & & $\mathrm{B}=0 \mathrm{~T}$ & $\mathrm{~B}=1 \mathrm{~T}$ & $\mathrm{~B}=0 \mathrm{~T}$ & $\mathrm{~B}=1 \mathrm{~T}$ & $\mathrm{~B}=0 \mathrm{~T}$ & $\mathrm{~B}=1 \mathrm{~T}$ \\
\hline $\mathrm{E}=97.4 \mathrm{MeV}$ & 97.4 & 0 & 0 & 99.9 & 99.7 & 0.2 & 0.2 \\
\hline $\mathrm{E}=148.2 \mathrm{MeV}$ & 148.2 & 0 & 0 & 99.9 & 100.0 & 0.2 & 0.2 \\
\hline $\mathrm{E}=188.7 \mathrm{MeV}$ & 188.7 & 0 & 0 & 100.0 & 100.0 & 0.3 & 0.2 \\
\hline Box4@60 mm & $72.4-105.4$ & 0 & 0 & 99.5 & 96.2 & 0.3 & 0.4 \\
\hline Box4@100 mm & $105.4-132.0$ & -0.2 & 0 & 99.8 & 97.9 & 0.3 & 0.4 \\
\hline Box4@140 mm & $130.8-155.8$ & -0.3 & 0 & 98.4 & 98.0 & 0.3 & 0.4 \\
\hline Box4@180 mm & $154.2-176.2$ & -0.3 & 0 & 98.4 & 96.3 & 0.4 & 0.4 \\
\hline
\end{tabular}



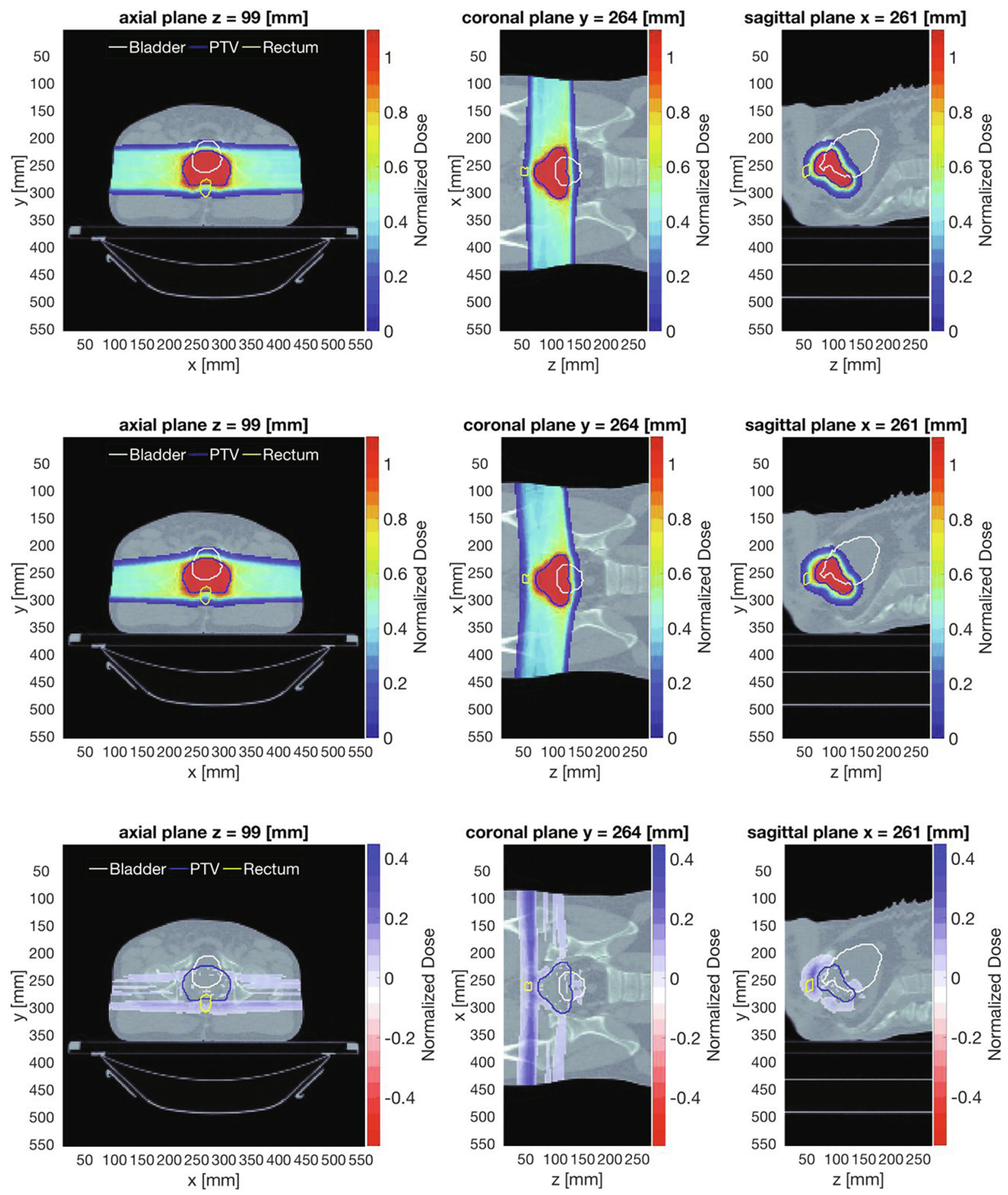

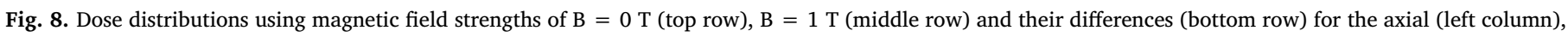

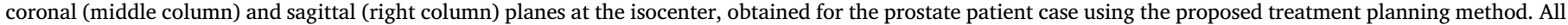
the results are normalized to the PTV prescribed dose of $68 \mathrm{~Gy}$. The magnetic field was applied transverse to the beam incident direction, alongside the $y$ axes.

chamber in magnetic fields of $\mathrm{B}=0 \mathrm{~T}$ and $\mathrm{B}=1 \mathrm{~T}$. The influence of the magnetic field on the intrinsic response of the Roos chamber was investigated for magnetic field strengths of 0 and $1 \mathrm{~T}$, before laterally integrated in-depth absorbed dose measurements were done. The detector was placed at a water equivalent depth of $20 \mathrm{~mm}$, transverse to a $100 \times 80 \mathrm{~mm}^{2}$ irradiation field. No statistically significant deviations, using a p-value of 0.05 , were observed between the chamber readings at $\mathrm{B}=0 \mathrm{~T}$ and $\mathrm{B}=1 \mathrm{~T}$ for our setup-specific configuration, i.e. the results were within the limits of our experimental uncertainties $(\sim 0.5 \%)$.
However, more detailed studies are required to analyze the impact of magnetic fields on the response of ionization chambers used in particle therapy as conventional dosimetric detectors. The experimental validation of the proposed dose calculation algorithm was technically limited due to constraints in our measurement setup. The extension of the current dosimetric procedures to our experimental conditions, using magnetic field environments, turned out to be very challenging. The performance of dosimetric equipment, such as conventional ionization chambers, diode and/or micro-diamond detectors within magnetic 

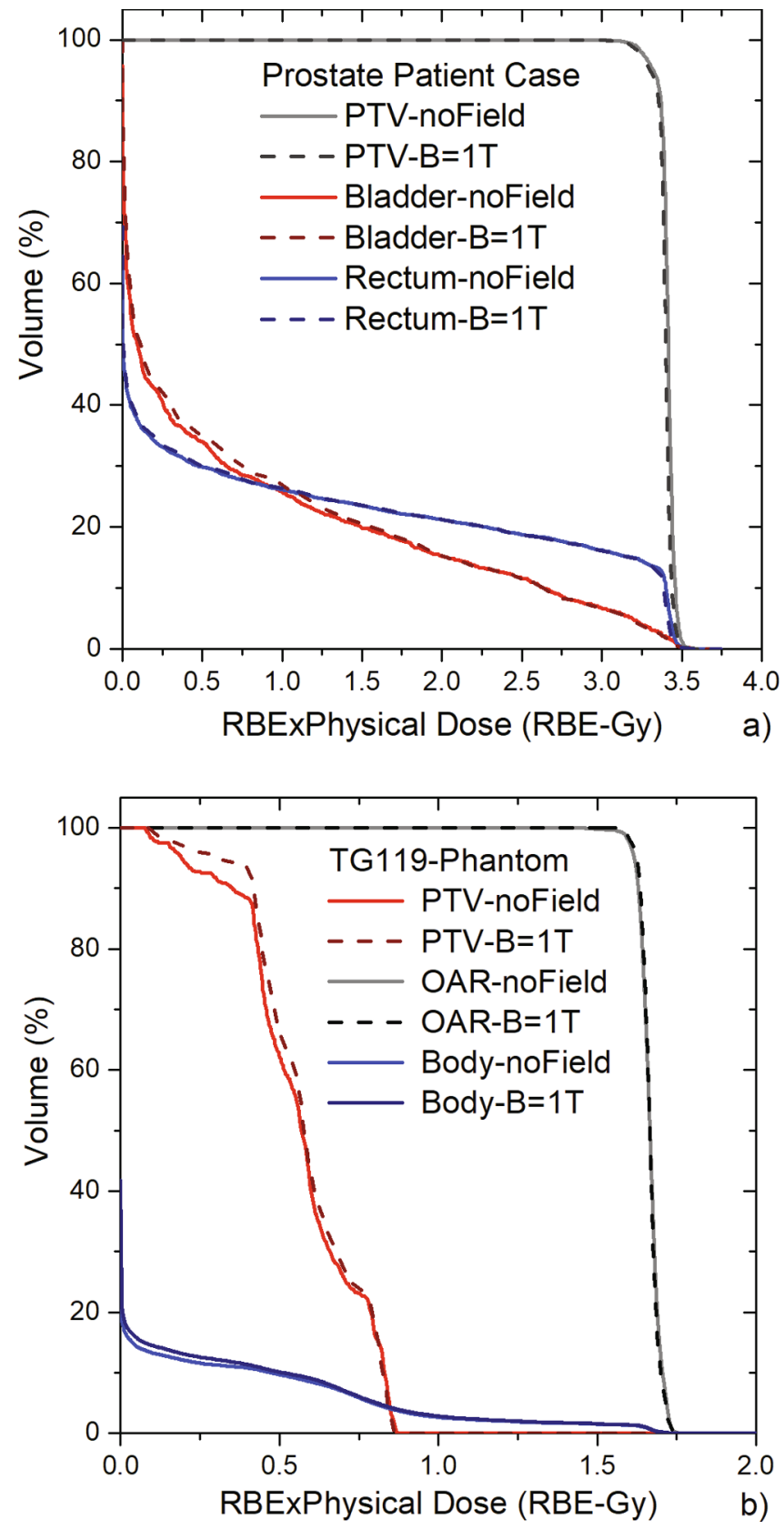

Fig. 9. DVH for the prostate patient (a) and the TG119 phantom (b) cases analyzed for plans calculated at $\mathrm{B}=0 \mathrm{~T}$ (solid lines) and $\mathrm{B}=1 \mathrm{~T}$ (dashed lines). RBE weighted physical doses, per treatment fraction, are displayed in the horizontal axis. fields are still not investigated and described for particle beams. In addition, due to geometrical constraints for our water phantom, the use of an array of detectors to perform multiple measurements for treatment plan verifications was not possible. Therefore, only in-depth dose distributions were acquired to validate the absolute values for absorbed doses. Current work is being performed to increase the dosimetric capabilities of our water phantom, with the design of specific chamber holders and more accurate positioning within the magnet. Measurements of lateral dose profiles are works in progress and certainly envisaged to complete the beam model validation process.

The dose calculation algorithm proposed through this work, relies on the accuracy of the MC beam model to describe proton interactions with realistic magnetic fields, as present on typical MRI scanners. The previous benchmarking of the model using reference measurements for in-depth and lateral profiles $[42,51,55,56]$ is therefore mandatory to ensure accurate dose estimations, as well as the description of the magnetic field maps as close as possible to reality. The proposed methodology is anticipated to be valid for different beam lines or magnetic field maps, as long as the calibration library data is replaced with custom IDD and lateral parameters.

\section{Conclusions}

An algorithm for treatment planning of protons in magnetic field regions was proposed using a custom dose calculation engine based on pencil beam kernels and MC simulated look up tables. The algorithm was successfully validated in water against MC recalculations and experimental data, using both single beams and SOBP.

The combination of a simple compensation method for correcting beam spot positions and the modification of the intrinsic dose engine allowed to create comparable treatment plans for the exemplary cases analyzed in this work, independently of the magnetic field strength. New adaptation methods for beam spot repositioning and more complex treatment planning studies are foreseen to establish the limits of applicability of the proposed method, especially when oblique angles are applied.

\section{Declaration of Competing Interest}

The authors declare that they have no known competing financial interests or personal relationships that could have appeared to influence the work reported in this paper.

\section{Acknowledgments}

The help and advices from Barbara Knäusl on treatment planning for this study are gratefully valued. The support of the MOCCAMED group providing access to their cluster and making possible all the MC simulations and evaluations in reasonable time is deeply appreciated. The financial support by the Federal Ministry of Science, Research and Economy and the National Foundation for Research, Technology and

Table 4

DVH parameters for the PTV and some OAR calculated for the TG119 phantom and the prostate patient cases for magnetic field intensities of 0 and 1 T.

\begin{tabular}{|c|c|c|c|c|c|c|c|}
\hline Case & Organ & B Field (T) & $\mathrm{D}_{2 \%}$ (Gy) & $\mathrm{D}_{50 \%}(\mathrm{~Gy})$ & $\mathrm{D}_{98 \%}(\mathrm{~Gy})$ & $\mathrm{V}_{95 \%}(\%)$ & $\mathrm{V}_{105 \%}(\%)$ \\
\hline \multirow[t]{6}{*}{ Prostate } & PTV & 0 & 3.46 & 3.40 & 3.23 & 0.98 & 0 \\
\hline & & 1 & 3.47 & 3.45 & 3.23 & 0.98 & 0 \\
\hline & Bladder & 0 & 3.43 & $<0.01$ & 0 & & \\
\hline & & 1 & 3.43 & $<0.01$ & 0 & & \\
\hline & Rectum & 0 & 3.38 & 0.09 & 0 & & \\
\hline & & 1 & 3.39 & 0.12 & $<0.01$ & & \\
\hline \multirow[t]{4}{*}{ TG119 } & PTV & 0 & 1.73 & 1.67 & 1.60 & 0.99 & $<0.01$ \\
\hline & & 1 & 1.73 & 1.67 & 1.61 & 1.00 & $<0.01$ \\
\hline & Spare OAR & 0 & 0.86 & 0.57 & 0.10 & & \\
\hline & & 1 & 0.85 & 0.58 & 0.15 & & \\
\hline
\end{tabular}


Development and the Austrian Science Fund (FWF) Project P 30065B27: Magnetic resonance imaging guided proton therapy (MAGIG-PRO) are gratefully acknowledged.

\section{References}

[1] Paganelli C, Whelan B, Peroni M, Summers P, Fast M, Van De Lindt T, et al. MRIguidance for motion management in external beam radiotherapy: current status and future challenges. Phys Med Biol 2018;63. https://doi.org/10.1088/1361-6560/ aaebcf.

[2] Winkel D, Bol GH, Kroon PS, van Asselen B, Hackett SS, Werensteijn-Honingh AM, et al. Adaptive radiotherapy: The Elekta Unity MR-linac concept. Clin Transl Radiat Oncol 2019;18:54-9. https://doi.org/10.1016/j.ctro.2019.04.001.

[3] Klüter S. Technical design and concept of a 0.35 T MR-Linac. Clin Transl Radiat Oncol 2019;18:98-101. https://doi.org/10.1016/j.ctro.2019.04.007.

[4] Lagendijk JJW, Raaymakers BW, Van den Berg CAT, Moerland MA, Philippens ME, van Vulpen M. MR guidance in radiotherapy. Phys Med Biol 2014;59:R349. https:// doi.org/10.1088/0031-9155/59/21/R349.

[5] Mittauer K, Paliwal B, Hill P, Bayouth JE, Geurts MW, Baschnagel AM, et al. A new era of image guidance with magnetic resonance-guided radiation therapy for abdominal and thoracic malignancies. Cureus 2018;10. https://doi.org/10.7759/ cureus.2422.

[6] Murray J, Tree AC. Prostate cancer - Advantages and disadvantages of MR-guided RT. Clin Transl Radiat Oncol 2019;18:68-73. https://doi.org/10.1016/j.ctro.2019. 03.006.

[7] Raaymakers BW, Jürgenliemk-Schulz IM, Bol GH, Glitzner M, Kotte ANTJ, Van Asselen B, et al. First patients treated with a 1.5 T MRI-Linac: Clinical proof of concept of a high-precision, high-field MRI guided radiotherapy treatment. Phys Med Biol 2017;62:L41-50. https://doi.org/10.1088/1361-6560/aa9517.

[8] Tetar SU, Bruynzeel AME, Lagerwaard FJ, Slotman BJ, Bohoudi O, Palacios MA. Clinical implementation of magnetic resonance imaging guided adaptive radiotherapy for localized prostate cancer. Phys Imaging Radiat Oncol 2019;9:69-76. https://doi.org/10.1016/j.phro.2019.02.002

[9] Hoffmann A. Integration of Magnetic Resonance Imaging and Proton Therapy n.d.

[10] Oborn BM, Dowdell S, Metcalfe PE, Crozier S, Keall PJ. Future of medical physics : Real-time MRI-guided proton therapy. Med Phys 2017;44:e77-90.

[11] Hartman J, Kontaxis C, Bol GH, Frank SJ, Lagendijk JJW, van Vulpen M, et al. Dosimetric feasibility of intensity modulated proton therapy in a transverse magnetic field of 1.5 T. Phys Med Biol 2015;60:5955-69. https://doi.org/10.1088/ 0031-9155/60/15/5955.

[12] Moteabbed M, Schuemann J, Paganetti H. Dosimetric feasibility of real-time MRIguided proton therapy. Med Phys 2014;41:111713https://doi.org/10.1118/1. 4897570.

[13] Raaymakers BW. Raaijmakers a JE, Lagendijk JJW. Feasibility of MRI guided proton therapy: magnetic field dose effects. Phys Med Biol 2008;53:5615-22. https://doi.org/10.1118/1.2962128.

[14] Schellhammer SM, Hoffmann AL, Gantz S, Smeets J, Van Der Kraaij E, Quets S, et al. Integrating a low-field open MR scanner with a static proton research beam line: Proof of concept. Phys Med Biol 2018;63.. https://doi.org/10.1088/1361-6560/ aaece8.

[15] Fuchs H, Moser P, Gröschl M, Georg D. Magnetic field effects on particle beams and their implications for dose calculation in MR-guided particle therapy. Med Phys 2017;44(3):1149-56. https://doi.org/10.1002/mp.12105.

[16] Oborn BM, Dowdell S, Metcalfe PE, Crozier S, Mohan R, Keall PJ. Proton beam deflection in MRI fields: Implications for MRI-guided proton therapy. Med Phys 2015;42:2113-24. https://doi.org/10.1118/1.4916661.

[17] Schellhammer SM, Hoffmann AL. Prediction and compensation of magnetic beam deflection in MR-integrated proton therapy: A method optimized regarding accuracy, versatility and speed. Phys Med Biol 2017;62:1548-64. https://doi.org/10. 1088/1361-6560/62/4/1548.

[18] Padilla-Cabal F, Georg D, Fuchs H. A pencil beam algorithm for magnetic resonance image-guided proton therapy. Med Phys 2018. https://doi.org/10.1002/mp.12854.

[19] Santos DM, Wachowicz K, Burke B, Fallone BG. Proton beam behavior in a parallel configured MRI-proton therapy hybrid: Effects of time-varying gradient magnetic fields. Med Phys 2018. https://doi.org/10.1002/mp.13309.

[20] Wolf R, Bortfeld T. An analytical solution to proton Bragg peak deflection in a magnetic field. Phys Med Biol 2012;57:N329-37. https://doi.org/10.1088/0031 9155/57/17/N329.

[21] Kurz C, Landry G, Resch AF, Dedes G, Kamp F, Ganswindt U, et al. A Monte-Carlo study to assess the effect of $1.5 \mathrm{~T}$ magnetic fields on the overall robustness of pencilbeam scanning proton radiotherapy plans for prostate cancer. Phys Med Biol 2017;62:8470-82. https://doi.org/10.1088/1361-6560/aa8de9.

[22] Burigo L and OB. MRI-guided proton therapy planning: accounting for an inline MRI fringe field. Phys Med Biol 2019; In press.

[23] Saini J, Maes D, Egan A, Bowen SR, St James S, Janson M, et al. Dosimetric evaluation of a commercial proton spot scanning Monte-Carlo dose algorithm: Comparisons against measurements and simulations. Phys Med Biol 2017;62:7659-81. https://doi.org/10.1088/1361-6560/aa82a5.

[24] Maes D, Saini J, Zeng J, Rengan R, Wong T, Bowen SR. Advanced proton beam dosimetry part II: Monte Carlo vs. pencil beam-based planning for lung cancer. Transl Lung. Cancer Res 2018;7:114-21.

[25] Wieser HP, Cisternas E, Wahl N, Ulrich S, Stadler A, Mescher H, et al. Development of the open-source dose calculation and optimization toolkit matRad. Med Phys 2017;44:2556-68. https://doi.org/10.1002/mp.12251.
[26] Hong L, Goitein M, Bucciolini M, Comiskey R, Gottschalk B, Rosenthal S, et al. A pencil beam algorithm for proton dose calculations. Phys Med Biol 1996. https:// doi.org/10.1088/0031-9155/41/8/005.

[27] Padilla-Cabal F, Fragoso J, Resch AF, Georg D, Fuchs H. Benchmarking a GATE/ Geant4 Monte Carlo model for proton beams in magnetic fields. Med Phys 2019. submitted.

[28] Resch AF, Elia A, Fuchs H, Carlino A, Stock M. Evaluation of electromagnetic and nuclear scattering models in GATE / Geant4 for proton therapy. Med Phys 2019. https://doi.org/10.1002/mp.13472.

[29] Elia A. Characterization of the GATE Monte Carlo platform for non isocentric treatments. 2019

[30] Ströbele J, Schreiner T, Fuchs H, Georg D. Comparison of basic features of proton and helium ion pencil beams in water using GATE. Z Med Phys 2012;22:170-8. https://doi.org/10.1016/j.zemedi.2011.12.001.

[31] Fuchs H, Vatnitsky S, Stock M, Georg D, Grevillot L. Nuclear Instruments and Methods in Physics Research B Evaluation of GATE / Geant4 multiple Coulomb scattering algorithms for a $160 \mathrm{MeV}$ proton beam 2017;410:122-6. https://doi.org/ 10.1016/j.nimb.2017.08.006.

[32] Jan S, Benoit D, Becheva E, Carlier T, Cassol F, Descourt P, et al. GATE V6: a major enhancement of the GATE simulation platform enabling modelling of CT and radiotherapy. Phys Med Biol 2011;56:881-901. https://doi.org/10.1088/0031 9155/56/4/001.

[33] Grevillot L, Frisson T, Zahra N, Bertrand D, Stichelbaut F, Freud N, et al. Optimization of GEANT4 settings for Proton Pencil Beam Scanning simulations using GATE. Nucl Instruments Methods Phys Res Sect B Beam Interact with Mater Atoms 2010;268:3295-305. https://doi.org/10.1016/j.nimb.2010.07.011.

[34] NIST. PSTAR database n.d.:http://physics.nist.gov/PhysRefData/Star/Text/PSTA.

[35] Frühwirth R, Regler M. On the quantitative modelling of core and tails of multiple scattering by Gaussian mixtures. Nucl Instruments Methods Phys Res Sect A Accel Spectrometers, Detect Assoc Equip 2001;456:369-89. https://doi.org/10.1016/ S0168-9002(00)00589-1.

[36] Gottschalk B, Cascio EW, Daartz J, Wagner MS. On the nuclear halo of a proton pencil beam stopping in water. Phys Med Biol 2015;60:5627-54. https://doi.org/ 10.1088/0031-9155/60/14/5627.

[37] Craft D, Bangert M, Long T, Papp D, Unkelbach J. Shared data for intensity modulated radiation therapy (IMRT) optimization research: The CORT dataset. GigaScience 2014;3:1-12. https://doi.org/10.1186/2047-217X-3-37.

[38] da Silva J, Ansorge R, Jena R. Fast pencil beam dose calculation for proton therapy using a double-gaussian beam model. Front Oncol 2015;5:281. https://doi.org/10 3389/fonc. 2015.00281

[39] Egashira Y, Nishio T, Hotta K, Kohno R, Uesaka M. Application of the pencil-beam redefinition algorithm in heterogeneous media for proton beam therapy. Phys Med Biol 2013;58:1169-84. https://doi.org/10.1088/0031-9155/58/4/1169.

[40] Soukup M, Fippel M, Alber M. A pencil beam algorithm for intensity modulated proton therapy derived from Monte Carlo simulations. Phys Med Biol 2005;50:5089-104. https://doi.org/10.1088/0031-9155/50/21/010.

[41] Saini J, Traneus E, Maes D, Regmi R, Bowen SR, Bloch C, et al. Advanced Proton Beam Dosimetry Part I: Review and performance evaluation of dose calculation algorithms. Transl Lung Cancer Res 2018;7:171-9https://doi.org/10.21037/tlcr. 2018.04.05.

[42] Parodi K, Mairani A, Brons S, Hasch BG, Sommerer F, Naumann J, et al. Monte Carlo simulations to support start-up and treatment planning of scanned proton and carbon ion therapy at a synchrotron-based facility. Phys Med Biol 2012;57:3759-84. https://doi.org/10.1088/0031-9155/57/12/3759.

[43] Bellinzona VE, Ciocca M, Embriaco A, Fontana A, Mairani A, Mori M, et al. Physica Medica On the parametrization of lateral dose pro fi les in proton radiation therapy. Phys Medica 2015;31:484-92. https://doi.org/10.1016/j.ejmp.2015.05.004.

[44] Bellinzona EV, Ciocca M, Embriaco A, Ferrari A, Fontana A, Mairani A, et al. A model for the accurate computation of the lateral scattering of protons in water. Phys Med Biol 2016;61:N102-17. https://doi.org/10.1088/0031-9155/61/4/ N102.

[45] Parodi K, Mairani A, Sommerer F. Monte Carlo-based parametrization of the latera dose spread for clinical treatment planning of scanned proton and carbon ion beams. J Radiat Res 2013;54:91-6. https://doi.org/10.1093/jrr/rrt051.

[46] Pedroni E, Scheib S, Böhringer T, Coray A, Grossmann M, Lin S, et al. Experimental characterization and physical modelling of the dose distribution of scanned proton pencil beams. Phys Med Biol 2005;50:541-61. https://doi.org/10.1088/00319155/50/3/011.

[47] Haberer T, Brons S, Hasch BG, Sommerer F, Naumann J, Parodi K, et al. Monte Carlo simulations to support start-up and treatment planning of scanned proton and carbon ion therapy at a synchrotron-based facility. Phys Med Biol 2012;57:3759-84. https://doi.org/10.1088/0031-9155/57/12/3759.

[48] Sawakuchi GO, Titt U, Mirkovic D, Ciangaru G, Zhu XR, Sahoo N, et al. Monte Carlo investigation of the low-dose envelope from scanned proton pencil beams. Phys Med Biol 2010;55:711-21. https://doi.org/10.1088/0031-9155/55/3/011.

[49] Fuchs H, Ströbele J, Schreiner T, Hirtl A, Georg D. A pencil beam algorithm for helium ion beam therapy. Med Phys 2012;39:6726. https://doi.org/10.1118/1. 4757578.

[50] Rana S, Greco K, Samuel EJJ, Bennouna J. Radiobiological and dosimetric impact of RayStation pencil beam and Monte Carlo algorithms on intensity-modulated proton therapy breast cancer plans. J Appl Clin Med Phys 2019;20:36-46. https://doi.org/ 10.1002/acm2.12676.

[51] Kozłowska WS, Böhlen TT, Cuccagna C, Ferrari A, Fracchiolla F, Georg D, et al. FLUKA particle therapy tool for Monte Carlo independent calculation of scanned proton and carbon ion beam therapy. Phys Med Biol 2019. https://doi.org/10 1088/1361-6560/ab02cb. 
[52] Mairani A, Böhlen TT, Schiavi A, Tessonnier T, Molinelli S, Brons S, et al. A Monte Carlo-based treatment planning tool for proton therapy. Phys Med Biol 2013;58:2471-90. https://doi.org/10.1088/0031-9155/58/8/2471.

[53] Agostinelli S, Allison J, Amako K, Apostolakis J, Araujo H, Arce P, et al. Geant4-a simulation toolkit. Nucl Instruments Methods Phys Res Sect A Accel Spectrometers, Detect Assoc Equip 2003;506:250-303. https://doi.org/10.1016/S0168-9002(03) 01368-8.

[54] Ferrari A, Sala PR, Fassò A, Ranft J. FLUKA: A Multi-Particle Transport Code,
CERN-2005-010, INFN TC 05/11, SLAC-R-773 2005:406.

[55] Tessonnier T, Böhlen TT, Ceruti F, Ferrari A, Sala P, Brons S, et al. Dosimetric verification in water of a Monte Carlo treatment planning tool for proton, helium, carbon and oxygen ion beams at the Heidelberg Ion Beam Therapy Center. Phys Med Biol 2017;62:6579-94. https://doi.org/10.1088/1361-6560/aa7be4.

[56] Grevillot L, Bertrand D, Dessy F, Freud N, Sarrut D. A Monte Carlo pencil beam scanning model for proton treatment plan simulation using GATE/GEANT4. Phys Med Biol 2011;56:5203-19. https://doi.org/10.1088/0031-9155/56/16/008. 